Annales Geophysicae (2002) 20: 377-390 (C) European Geophysical Society 2002

\title{
Electrostatic interaction between Interball-2 and the ambient plasma. 2. Influence on the low energy ion measurements with Hyperboloid
}

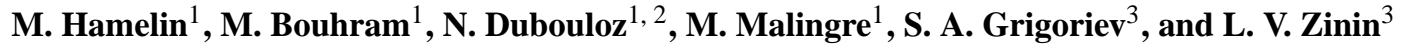 \\ ${ }^{1}$ Centre d'Etude des Environnements Terrestre et Planétaires, 4 av. de Neptune, 94107 Saint Maur, France \\ ${ }^{2}$ Laboratoire de Physique et Chimie de l'Environnement, 45071Orléans cedex, France \\ ${ }^{3}$ Kaliningrad State University, Mathematical Dept., A. Nevski ul. 14, 236041 Kaliningrad, Russia
}

Received: 2 March 2001 - Revised: 20 December 2001 - Accepted: 8 January 2002

\begin{abstract}
The measurement of the thermal ion distributions in space is always strongly influenced by the ion motion through the complex 3D electrostatic potential structure built around a charged spacecraft. In this work, we study the related aberrations of the ion distribution detected on board, with special application to the case of the Hyperboloid instrument borne by the Interball-2 auroral satellite. Most of the time, the Interball-2 high altitude auroral satellite is charged at some non-negligible positive potential with respect to the ambient plasma, as shown in part 1 ; in consequence, the measurement of magnetospheric low energy ions $(<80 \mathrm{eV})$ with the Hyperboloid instrument can be disturbed by the complex electric potential environment of the satellite. In the case of positive charging, as in previous experiments, a negative bias is applied to the Hyperboloid structure in order to reduce this effect and to keep as much as possible the opportunity to detect very low energy ions. Then, the ions reaching the Hyperboloid entrance windows would have travelled across a continuous huge electrostatic lens involving various spatial scales from $\sim 10 \mathrm{~cm}$ (detector radius) to $\sim 10 \mathrm{~m}$ (satellite antennas). Neglecting space charge effects, we have computed the ion trajectories that are able to reach the Hyperboloid windows within their acceptance angles. There are three main results: (i) for given values of the satellite potential, and for each direction of arrival (each window), we deduced the related energy cutoff; (ii) we found that all ions in the energy channel, including the cutoff, can come from a large range of directions in the unperturbed plasma, especially when the solar panels or antennas act as electrostatic mirrors; (iii) for higher energy channels, the disturbances are reduced to small angular shifts. Biasing of the aperture is not very effective with the Hyperboloid instrument (as on previous missions with instruments installed close to the spacecraft body) because the potential environment is driven by effects from the spacecraft. Our results are used to explain some unexpected features of the low energy ion mea-
\end{abstract}

Correspondence to: M. Hamelin

(michel.hamelin@cetp.ipsl.fr) surements, especially during polar wind events recorded by Hyperboloid. In conclusion, knowing the satellite potential from IESP measurements, we were able to reject any low energy doubtful data and to perform angular corrections for all higher energy ion data. Then the selected and corrected data are a reliable basis for interpretation and estimation of the thermal ion distributions.

Key words. Space plasma physics (charged particle motion and acceleration; numerical simulation studies; spacecraft sheaths, wakes, charging)

\section{Introduction}

Thermal ion measurements in the magnetosphere have always depended upon a precise understanding of the satellite charging. For a satellite orbiting at high altitudes in the magnetosphere, the satellite potential relative to the ambient plasma ranges typically from a few volts positive up to $50 \mathrm{~V}$ in the tail lobes (Pedersen, 1995). These values prevent the low energy ions from reaching the onboard spectrometers, and ions that have energies lower than the satellite potential are missed. Due to this problem, some potential control by applying a negative bias potential between the ion detectors and the satellite body were successfully tested on the GEOS 1, GEOS 2 (Décreau et al., 1978) and DE-1 (Olsen et al., 1986) satellites. However, a model of the potential distribution surrounding the Retarding Ion Mass Spectrometer (RIMS) aboard DE-1 (Olsen et al., 1986), neglecting space charge effects, indicated that if the spacecraft potential is more than few a volts positive, there can be an electrostatic potential barrier inhibiting the effectiveness of the aperture bias imposed on the instrument's surface. Conversely, ions with energies higher than the satellite potential can be detected, but the satellite potential value has to be known in order to correct the measured energies, while angular corrections of the measured distributions require an accurate knowledge of the three-dimensional potential dis- 

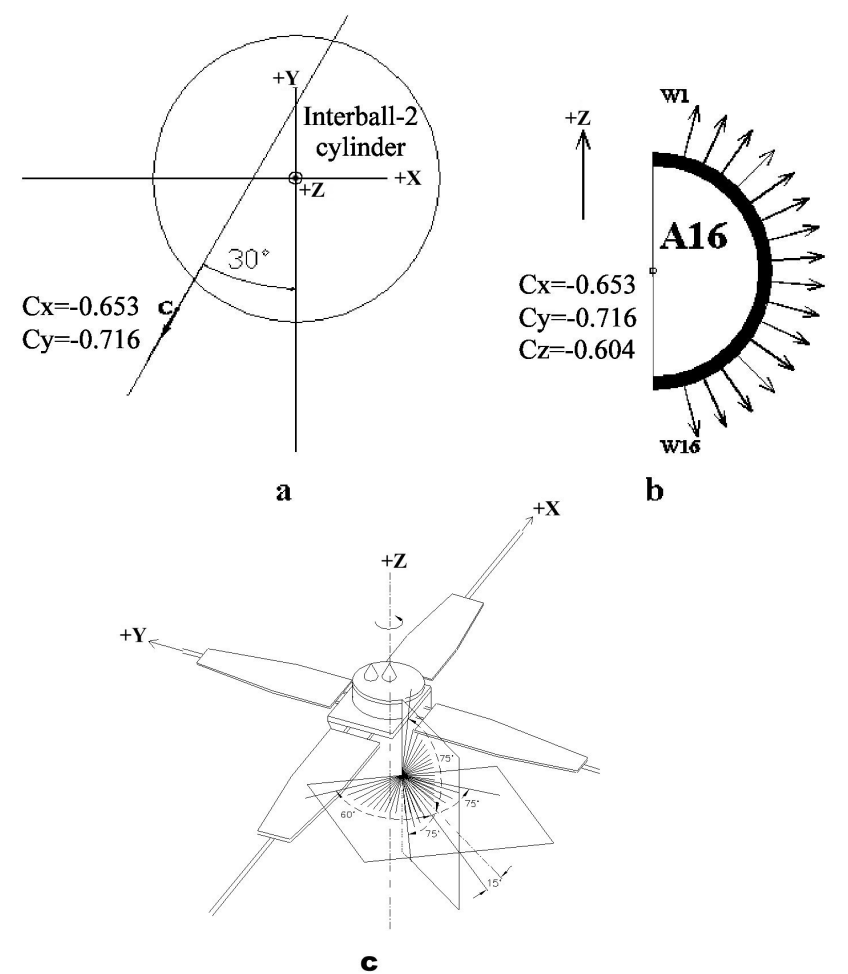

b

Fig. 1. Position and pointing directions of the Hyperboloid A16 analyser: (a) trace of the detector plane on the $X Y$ plane and A16 center position $\left(C_{x}, C_{y}, C_{z}\right)$, (b) cut showing pointing directions in the A16 detector plane, and (c) global view showing the geometry of the two analyzers relative to the satellite body.

tribution surrounding the satellite, particularly for a satellite with a complex geometric shape.

The Hyperboloid instrument (Girard et al., 1995; Dubouloz et al., 1998) aboard the Interball-2 satellite is an ion mass spectrometer measuring three-dimensional distributions of low energy ions $(<80 \mathrm{eV})$ at altitudes from 700 to $20000 \mathrm{~km}$, over the auroral zone. The biasing technique mentioned above has also been applied to Hyperboloid. The instrument has been designed to select the major ionic species: as a function of mass and charge $\left(\mathrm{H}^{+}, \mathrm{He}^{+}, \mathrm{O}^{+}\right.$and $\mathrm{O}^{++}$), as a function of several energy ranges and as a function of their direction of arrival through the multiple windows of its two analyzers, also taking advantage of the rotation of the satellite (Dubouloz et al., 1998). Statistical observations from electric field experiments aboard Interball-2 showed that at high altitudes (15000-20000 km), the spacecraft body potential reaches positive values ranging from 0 to $12 \mathrm{~V}$ (Torkar et al., 1999). Since the energy of the measured ions is comparable to the typical spacecraft potentials, a precise knowledge of both spacecraft body potential and the three-dimensional potential distribution surrounding the body is required for correcting the ion distributions.

For the low plasma densities encountered at high altitudes on the Interball-2 orbit, the space charge has a minor influence to the potential structure around the spacecraft. The

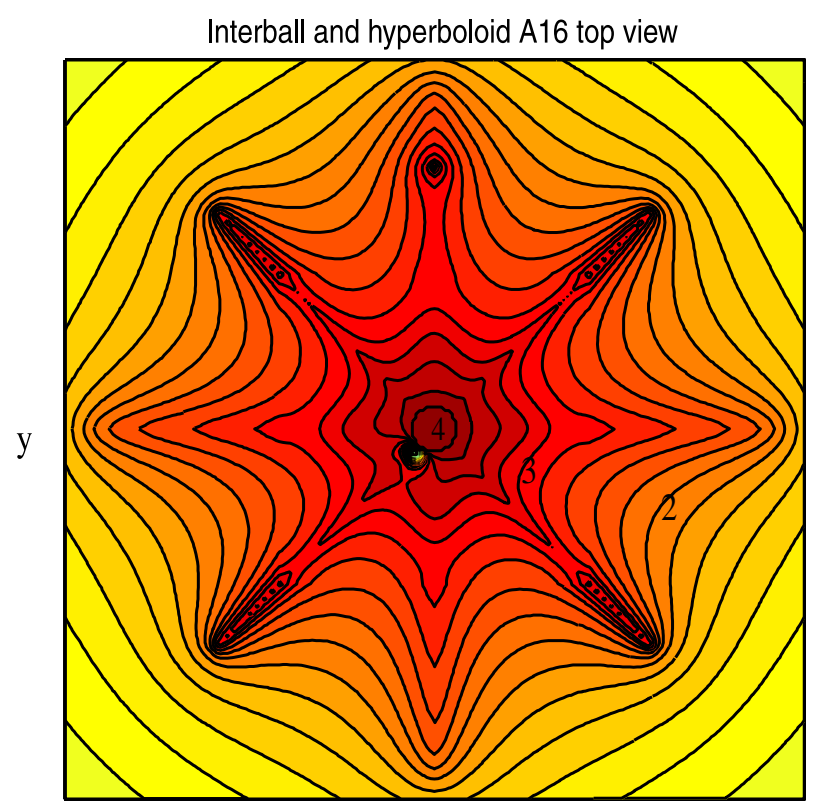

$\mathrm{x}$

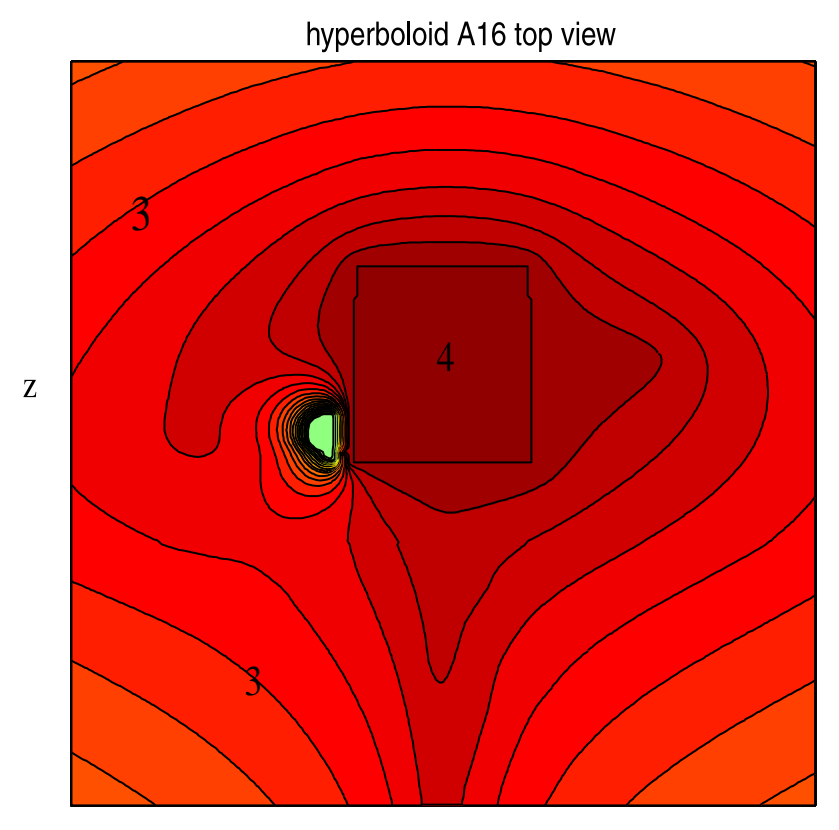

$\mathrm{X}$

Fig. 2. Equipotential contours in the A16 detector plane for $\Phi_{h}=$ $-4 \mathrm{~V}, \Phi_{s}=4 \mathrm{~V}$ in: (a) the $6 \times 6 \mathrm{~m}$ section in the A16 plane, and (b) the $24 \times 24 \mathrm{~m}$ section in the $X Y$ plane at $Z=Z_{C}$ (center of A16). A color scale is displayed, starting from brown for $+4 \mathrm{~V}$ to green for $-4 \mathrm{~V}$ (contour step: $0.2 \mathrm{~V}$ ).

infinite Debye length assumption is suitable for first order calculations and then, we will consider that the potential is the solution of the Laplace equation with the spacecraft at a potential inferred from IESP electric field double-probe measurements and theoretical considerations (see the companion paper by Bouhram et al., 2001).

The major practical difficulty to compute the ion trajecto- 


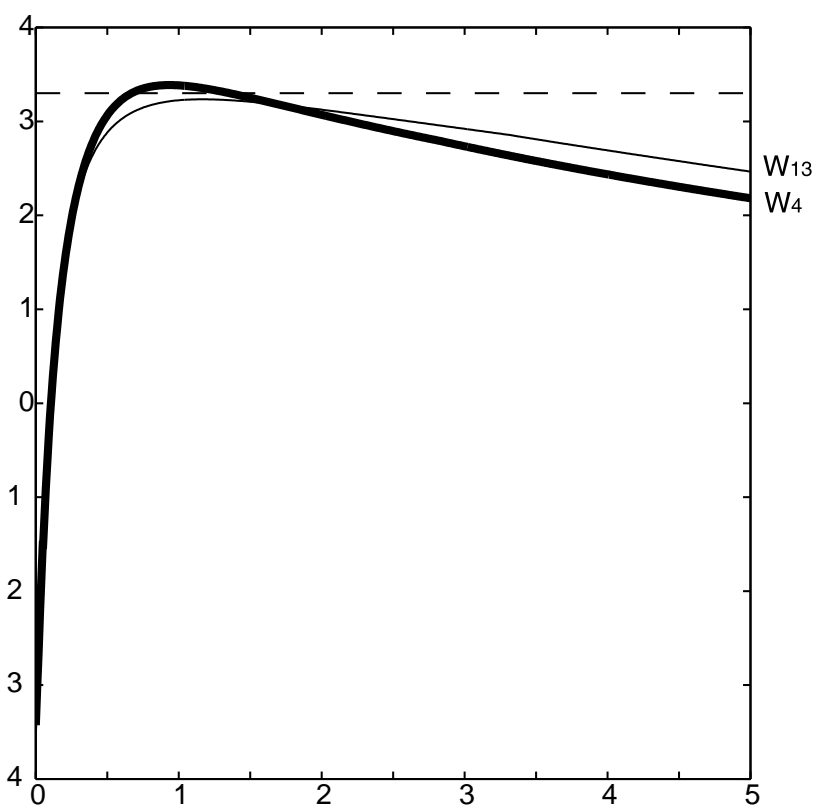

Fig. 3. Curves of the potential versus distance along the directions of windows $w_{4}$ (thick curve) and $w_{13}$ (thin curve).

ries is to manage the various spatial scales involved in the satellite description and then in the ion motion. First, the Hyperboloid windows are embedded in the two cylindrically shaped detectors (radius $15 \mathrm{~cm}$ and $10 \mathrm{~cm}$, (see Dubouloz et al., 1998, Fig. 1). We set the potential of the cylinders to a few volts and the electric field around the cylinders reaches about several tens of $\mathrm{V} / \mathrm{m}$ in the radial direction: this is a first electrostatic lens which bends the trajectories towards or outwards from the radial direction. A second lens with a lower field and a space scale of about $1 \mathrm{~m}$ takes place around the cylindrical body of the satellite; then for some particular directions, the solar panels, which have an extent of $\sim 3 \mathrm{~m}$, could either collect or reflect the ions. Finally, the nine thin and $\sim 10 \mathrm{~m}$ long antennas extend the satellite equipotential very far from the satellite body itself (Fig. 2 of Bouhram et al., 2001).

A first task has been to improve both the geometrical description of the Interball-2 satellite, including the Hyperboloid instrument, with a thin resolution and to increase the precision of the calculations of the potential in 3 dimensions. Single backward trajectories are computed, starting from the individual Hyperboloid windows: the ions can (i) return into the detector because of their energy that is too low, which means that these ions should never be observed, (ii) hit the satellite body or any solar panel or antenna (then these items shadow the detector windows and again, these ions should be never observed), (iii) cross the external border of our computational domain, a $30 \mathrm{~m}$ radius sphere: the inverse trajectories can then be those of magnetospheric thermal ions detected by Hyperboloid. Of course, only type (iii) trajectories correspond to real trajectories of plasma ions able to hit the detector.

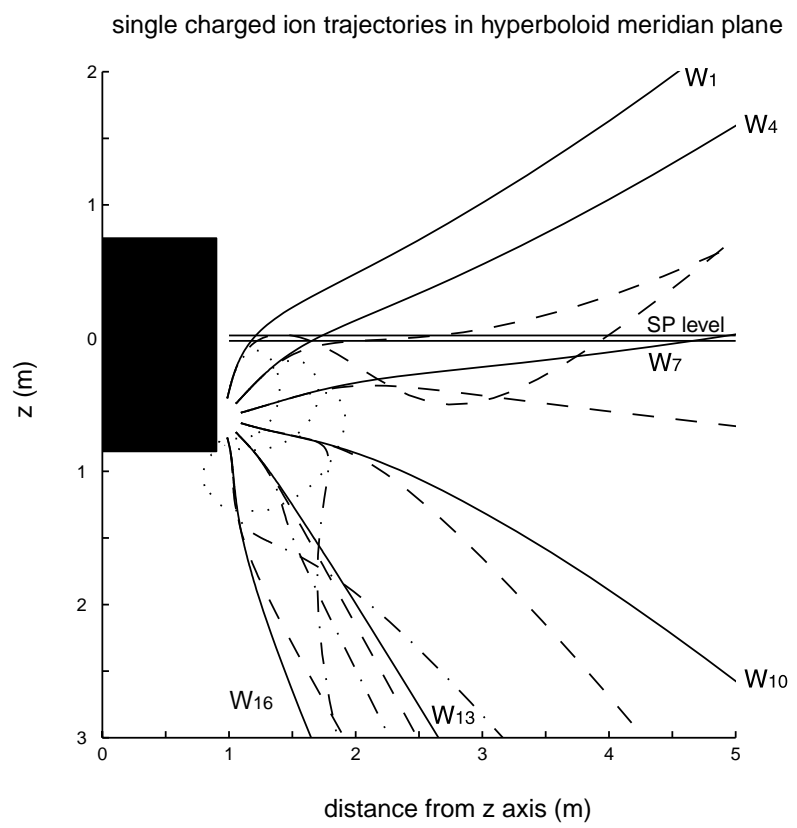

Fig. 4. Trajectories of single charged ions in the A16 detector plane $\left(\Phi_{h}=-4 \mathrm{~V}\right.$ and $\left.\Phi_{s}=+4 \mathrm{~V}\right)$ for different arrival energies: $8.01 \mathrm{eV}$ (solid line), $7.67 \mathrm{eV}$ (dashed line) and $7.33 \mathrm{eV}$ (dashdot line) and different arrival windows: $w_{1}, w_{4}, w_{7}, w_{10}, w_{13}$, and $w_{16}$.

The next part of this work is a statistical study based on multiple trajectories of ions that can enter the instrument. As a result, we obtain the probability of an ion to come from some direction (in the satellite frame). As the energy is conservative, the energy range is obviously just shifted by the Hyperboloid potential relative to the undisturbed plasma. Then, we apply our results to some polar wind data showing unexpected angular characteristics. According to our calculations, this appears to be due to the bending of ion trajectories in the complex satellite environment. Finally, we discuss the improvements to include in the data analysis and to foresee future experiment setups.

\section{Models and ion trajectories}

\subsection{Satellite model}

The Interball-2 structure is described in Bouhram et al. (2001) and in several previous papers (Dubouloz et al., 1998; Zinin et al., 1995, 1998). Here, for consistency, we review the parts of the present model, which are adapted for the purpose of ion trajectories reconstruction; we used the mechanical drawings provided by Mulyarchik (private communication)

The satellite model is built around a cylinder (diameter $0.75 \mathrm{~m}$, height $1.60 \mathrm{~m}$ ): we take the $Z$ axis as the cylinder axis. The satellite rotates around the $Z$ axis. There are 4 flat solar panels as in the $X Y$ plane (Fig. 1c) and $7.5 \mathrm{~m}$ booms at their end (the solar panels look in the $+Z$ direc- 
Table 1. Characteristics of the computational grids

\begin{tabular}{|c|c|c|c|c|c|c|c|}
\hline \multirow[t]{2}{*}{ Grid } & \multicolumn{3}{|c|}{ index range } & \multicolumn{3}{|c|}{ spatial range $(\mathrm{m})$} & \multirow[t]{2}{*}{ step (m) } \\
\hline & $\mathrm{nx}$ & ny & $\mathrm{nz}$ & $\mathrm{x}$ & $\mathrm{y}$ & $\mathrm{z}$ & \\
\hline$\Sigma$ & \pm 80 & \pm 80 & \pm 60 & \pm 40.0 & \pm 40.0 & \pm 30.0 & 0.500 \\
\hline$\Omega$ & \pm 52 & \pm 52 & \pm 52 & \pm 13.0 & \pm 13.0 & \pm 13.0 & 0.250 \\
\hline$\omega$ & \pm 200 & \pm 200 & \pm 60 & \pm 5.0 & \pm 5.0 & \pm 1.5 & 0.025 \\
\hline
\end{tabular}

tion). The satellite cylinder lies between $Z=-0.850 \mathrm{~m}$ and $Z=+0.75 \mathrm{~m}$. Four other $10 \mathrm{~m}$ antennas at $45^{\circ}$ from the first set of 4 are in a $X Y$ plane at $Z=-0.75 \mathrm{~m}$. A long antenna stands along the $-Z$ axis. A spherical propellant tank close to Hyperboloid has also been included in the model (diameter $0.30 \mathrm{~m}$, center: $-0.335,-0.810,-0.250 \mathrm{~m}$ ).

An accurate description of such a large structure requires the use of several grids, as in the previous calculations (Zinin et al., 1995, 1998). Hopefully, the availability of larger and faster computing facilities will allow us to use one larger and finer inner grid embedding the satellite and the solar panels instead of 5 separate grids as in this previous work. We also extended the medium grid and the larger extent coarser grid. The characteristics of the grids are summarised in Table 1: the satellite, Hyperboloid and the solar panels are described in the $\omega$ grid with $2.5 \mathrm{~cm}$ spacing. The medium grid $\Omega$ with a $12.5 \mathrm{~cm}$ spatial resolution includes all long antennas except the $-Z$ one, and the outer grid $\Sigma$ extends the calculation domain to $\pm 40.0 \mathrm{~m}$ in $X Y$ and to $\pm 30.0 \mathrm{~m}$ along $Z$.

\subsection{Hyperboloid model}

The Hyperboloid instrument has been modeled as a series of simple geometrical volumes: rectangular boxes and cylinders representing the housing of the two analyzers A10 and A16 with, respectively, 10 and 16 windows (see Dubouloz et al., 1998). The potential of the grid points in the Hyperboloid volume are set to a potential value biased with respect to the satellite body as in reality. We can then derive the complete potential map $\Phi(x, y, z)$ solving the Laplace equation by finite difference schemes. The window centers have been used as the inverse starting points of the ion trajectories calculations. We show in Fig. 1a the trace of the A16 analyzer plane which is parallel to the $Z$ axis. The pointing directions of all $\mathrm{A} 16$ windows $w_{i}$ are issued from point $\mathrm{C}$ with the angular directions in the analyzer plane shown in Fig. $1 \mathrm{~b}$. The $\theta_{i}$ polar angle directions with respect to the $Z$ axis are $15^{\circ}, 25^{\circ}, \ldots 165^{\circ}$. The A10 analyzer plane is perpendicular to the A16 plane and parallel to the pointing direction of window $w_{10}$ (at $105^{\circ}$ from the $Z$ axis). We do not consider the A10 analyzer in this paper; therefore, all windows $w_{i}$ will refer to those of the A16 analyzer. In fact, each window selects ions in a $10^{\circ} \times 10^{\circ}$ angular field of view around the central directions $\left(\theta_{i}, \phi_{i}\right)$, where $\phi_{i}$ is the azimuthal angle. The ions are also selected as a function of energy in several steps around the central values $E_{i}$. For the basic mode that we consider here, there are 17 steps $\left[E_{a i}-E_{b i}\right]$ listed in Table 2 (for the full list of modes, see Table 1 in Dubouloz et al., 1998). We used contiguous square energy windows $\left(E_{b i}=E_{a i+1}\right)$ in our model. This assumption is justified as the acceptance of the analyzers drops rapidly outside the energy steps, but we shall keep in mind for data analysis that the energy limits are not so sharp in reality than they would appear in our simulations.

\subsection{Potential maps and interpolation}

In theory, in order to deduce any potential map driven by the potential of the satellite $\left(\Phi_{s}\right)$ and the detector potential $\left(\Phi_{h}\right)$, we can merge two maps: one with $u_{s}=1$ and $u_{h}=0$, the other one with $u_{s}=0$ and $u_{h}=1$ ( $u$ is the potential normalized to the potential of the unique conductor with nonzero potential). In practice, for numerical reasons, we set $u_{h}=0.001$ instead of 0 , introducing a negligible energy shift of less than $10 \mathrm{meV}$ on the results. The relative accuracy of the trajectories' calculations remains high, whereas the problem itself has been modified by a negligible energy shift.

The normalized potential maps $u(x, y, z)$ are calculated by the same Laplace multi-grid solver as described in Zinin et al. (1998), but now with an accuracy of $10^{-5}$ using larger floating numbers and finer grids. The increase in the accuracy was needed because the process of the derivation of the electric field by interpolation and the differences on the potential tables decreases the resulting accuracy; moreover, in the case of small variations of the potential, the finer grids could even give worse results than coarser ones.

The interpolation procedure was tested using the same set of grids with a spherical model of satellite by comparison of the interpolated values with the analytical potential and electric field. The usual 8 points, first order 3D interpolation scheme (weight of the 8 corners of a cube proportional to the sub-volumes defined from the point) gave insufficient accuracy, especially for the derived electric field. Then, we used a second order 11 points scheme, equivalent to the full 27 points, provided that the points of the grid fulfill the Laplace equation. Let us write the potential as:

$$
\begin{aligned}
\Phi= & a_{0}+a_{x} x+a_{y} y+a_{z} z+a_{x y} x y+\ldots \\
& +a_{x x y} x^{2} y+\ldots a_{x y z} x y z .
\end{aligned}
$$

All Laplace operators applied on terms with factors $x y, y z, x z$ and $x y z$ will give 0 . The Laplace operator ap- 
Table 2. Energy channels in eV

\begin{tabular}{cccccccccccccccccc}
\hline $\mathrm{i}$ & 1 & 2 & 3 & 4 & 5 & 6 & 7 & 8 & 9 & 10 & 11 & 12 & 13 & 14 & 15 & 16 & 17 \\
$E_{a i}$ & 1.31 & 1.89 & 2.33 & 2.93 & 3.68 & 4.62 & 5.81 & 7.26 & 9.04 & 11.3 & 14.1 & 17.7 & 22.1 & 27.7 & 34.6 & 43.2 & 54.0 \\
$E_{b i}$ & 1.89 & 2.33 & 2.93 & 3.68 & 4.62 & 5.81 & 7.26 & 9.04 & 11.3 & 14.1 & 17.7 & 22.1 & 27.7 & 34.6 & 43.2 & 54.0 & 67.4 \\
\hline
\end{tabular}

plied on a term as $x^{2} y$ would give a result proportional to $y$. This is not acceptable since it should be constant, and consequently, the corresponding coefficients shall be 0 . Then, the potential can be written as the sum of 11 terms:

$$
\begin{aligned}
\Phi= & a_{0}+a_{x} x+a_{y} y+a_{z} z+a_{x x} x^{2}+a_{y y} y^{2}+a_{z z} z^{2} \\
& +a_{x y} x y+a_{y z} y z+a_{x z} x z+a_{x y z} x y z .
\end{aligned}
$$

The 11 coefficients are derived from the values of the potential on 11 neighbours of the current point. For a given point, the procedure that we use is:

- select the closest grid point (1 grid point);

- select neighbours in $\pm x, \pm y, \pm z$ directions (6 grid points);

- select the small $2 \times 2 \times 2$ sub-cube which contains the point and use the 4 points which were not selected yet ( 3 grid points from the $3 \times 3 \times 3$ cube edge and 1 grid point from the cube apex).

Close to the borders of the computational grids, 1-step shifts in $x, y, z$ are made to find a $3 \times 3 \times 3$ cube in the computing domain. The potential and electric fields are deduced using the analytical interpolation expression of the potential. Then, with our grid system, the resulting relative accuracy for the electric field is about $\sim 10^{-4}$.

The electric field deduced from the Laplace solver, the merging procedure and the potential interpolation scheme is valid everywhere except in the close vicinity ( $\sim$ grid step) of the conductive bodies: this fact is inherent in the precision of the geometrical description of the bodies. Let us consider the A16 analyzer cylindrical structure, whose radius is $15 \mathrm{~cm}$ and is defined with a grid spacing of $2.5 \mathrm{~cm}$, charged at $10 \mathrm{~V}$ : if it was a sphere of that radius, the potential would decrease by $\sim 1.5 \mathrm{~V}$ at a single grid spacing distance from the structure (of the order of the first energy steps of the analyzer). This observation justifies the use of grid spacing as small as $2.5 \mathrm{~cm}$ and the use of an analytical description at shorter distances from the analyzer, the satellite or any conductive item. The approximate analytical derivation of the electric field close to the analyzer is based on the fact that from about a one-step distance from each detector window, the analyzers A10 and A16 look like cylinders. The cylinders' radius ( $15 \mathrm{~cm}$ for A16 which is the focus of this paper) is much larger than the step. The cylinders' extensions in the axis direction $(\sim+10 \mathrm{~cm}$ and $\sim-20 \mathrm{~cm}$ in the two sides for A16) are also much larger than the step. Consequently, we can describe the electric field in the first step range as the field between two infinite coaxial cylinders; one is the cylindrical analyzer cover set at the bias potential and the other is a fictitious cylinder at a close distance ( $\sim$ one step) whose potential is taken from the numerical solution of the Laplace equation which is valid at this distance. We use the value of the potential given by the grid interpolation at a point facing each analyzer window at a radial distance of $\sim \sqrt{ } 3$ steps, i.e. $\sim 4.3 \mathrm{~cm}$. The ion motion between the two cylinders is deduced analytically and the result is a secondary "inverse starting point" at a distance of the body where the numerical integration of the equations of motion can be performed accurately.

We show in Fig. 2 two cuts in the calculated 3D maps in a typical case for which the satellite is charged at $+4 \mathrm{~V}$ and Hyperboloid is set to $-4 \mathrm{~V}$ by the $\Phi_{\text {bias }}=8 \mathrm{~V}$ applied negative bias. The top panel is a cut perpendicular to the spin axis and is located at the $z$ level of the centre of the A16 analyzer. As Hyperboloid lies in the middle level between the solar panel antennas plane and the perpendicular antenna system at $45^{\circ}$ from these former antennas, the resulting influences build an 8 apex star figure along the antenna directions. Some convergence effect on the incoming ions can be expected. Close to Hyperboloid in the bottom-left direction, a saddle potential structure is present, which would produce focusing - defocusing effects on the ions. The bottom panel is a cut through a plane parallel to the A16 analyzer containing the spin axis $Z$ (see Fig. 1a). The potential on any straight line issued from the A16 analyzer windows rises from $-4 \mathrm{~V}$ to a maximum in the range $3.0-3.6 \mathrm{~V}$ depending on the direction considered, and then decreases towards $0 \mathrm{~V}$ at infinite distance. There is a slightly lower potential barrier in the $w_{12}$ to $w_{16}$ directions (bottom-left direction in the figure). The variation of potential in the A16 plane as a function of the distance from the windows in a straight central direction has been plotted in Fig. 3 in the cases of windows $w_{4}\left(\theta_{4}=45^{\circ}\right)$ and $w_{13}\left(\theta_{13}=135^{\circ}\right)$. The fact that the potential rises very rapidly to the maxima around $3.3 \mathrm{eV}$ within the range $0.5-1 \mathrm{~m}$ shows the importance of the external shape of the analyzer which acts as an electrostatic lens. The potential barrier is higher in the case of $w_{4}$ than in the case of $w_{10}$, but the difference between the two barriers is only $0.15 \mathrm{eV}$. Thus, it could induce only a very slight effect on the real measurements.

The two potential maps of Fig. 2 and potential curves of Fig. 3 are useful for illustrating the potential structure around the Interball-A satellite and the Hyperboloid analyzer, but they are $2 \mathrm{D}$ and $1 \mathrm{D}$ views of the potential. Of course, the trajectories, even for particles axially launched from the A16 windows, are 3D. Only the numerical calculation of these 

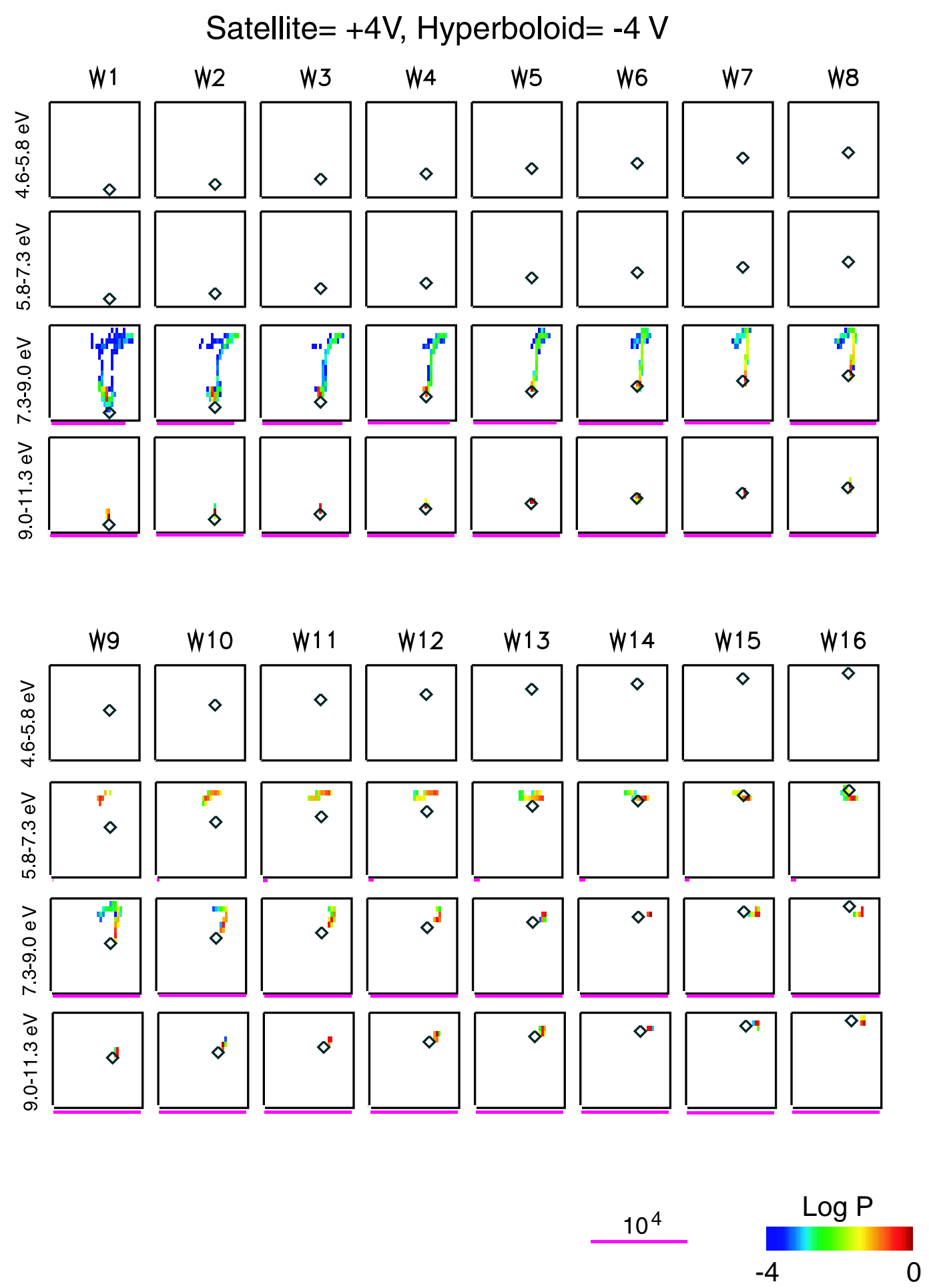

Fig. 5. Probabilities of incoming direction for a particle detected by a window and within an energy channel. The horizontal and vertical axes display the polar angle (top: $0^{\circ}$ to bottom $180^{\circ}$ ) and the azimuthal angle (left: $90^{\circ}$ to right: $360^{\circ}$ ), respectively, with an angular resolution of $10^{\circ}$. Diamonds indicate the viewing direction of each window. The pink bar below each plot represents the number of computer particles reaching the outer boundary of the simulation system normalised to the number of ions launched from each window. 
trajectories enables us to know the potential maxima reached along the trajectories.

One can see the relatively low efficiency of the biasing technique in this case which results in lowering the potential barrier by only $0.7 \mathrm{~V}$. Another remark is the complexity of the potential structure close to the satellite and particularly the lack of symmetry due to the position of Hyperboloid relatively to the 8 antenna system.

\subsection{Ion trajectories}

A single ion trajectory was retraced from the Hyperboloid's windows, solving the motion's equation:

$m_{i} \boldsymbol{r}=-q_{i} \nabla \Phi(r)$,

where $m_{i}$ and $q_{i}$ are the mass and charge of the ion, $r$ is the position and $\nabla V$ is the electrostatic potential gradient calculated as shown in Sect. 2.3. The magnetic field force has been neglected. For $1 \mathrm{eV} \mathrm{H}^{+}$and $\mathrm{O}^{+}$ions, the Larmor radius at the Interball-2 altitude is, respectively, about 100 and $500 \mathrm{~m}$, much larger than the $\sim 10 \mathrm{~m}$ satellite perturbed environment. However, incoming ions with energies close to the potential barrier height will be very sensitive to the magnetic force in the region near the potential maximum since they may cross this region with a very small velocity. In fact, we investigated more precisely the effect of such a magnetic field since there is no difficulty at all to add the magnetic additional term in the procedure and we will report about it later in the discussion of our results.

The integration scheme is a standard Runge-Kutta procedure with a variable step. The variation in the steps is controlled by several parameters: the required accuracy (controlled via the ion energy, which should be a constant of the motion), the grid spacing and the vicinity of conductive items. The approach of the conductive bodies is controlled through dual grids of booleans, indicating if a point is inside or outside the conductors.

We have choosen a common case in high altitude regions with the satellite at $\Phi_{s}=+4 \mathrm{~V}$ and the Hyperboloid analyzer at $\Phi_{h}=-4 \mathrm{~V}$ (the applied bias is $\Phi_{\text {bias }}=8 \mathrm{~V}$ ). We look at reverse trajectories of singly charged ions $\left(\mathrm{H}^{+}, \mathrm{He}^{+}\right.$ and $\mathrm{O}^{+}$) leaving the windows with the central direction, and with several energies (since the magnetic force is neglected, all single ions follow the same trajectory; the same calculation that we performed with the double charged ion $\mathrm{O}^{++}$ led to slightly different trajectories). For clarity, we reported in Fig. 4 the projection of the trajectories issued only from $w_{1}, w_{4}, w_{7}, w_{10}, w_{13}$ and $w_{16}$ on the A16 analyzer plane. For each window, we have chosen the same set of values for ion energies in the Hyperboloid frame close to the potential barrier: $7.33 \mathrm{eV}$ (dash-dot line), $7.67 \mathrm{eV}$ (dashed line) and $8.01 \mathrm{eV}$ (solid line). For $w_{1}, w_{4}$ and $w_{7}$, the trajectories with initial energies $7.33 \mathrm{eV}$ return to Hyperboloid (dotted lines) and so, they cannot correspond to trajectories of ions incoming from the plasma. Looking, in particular, at the $w_{1}$ trajectories, we see that the $7.67 \mathrm{eV}$ trajectory is first reflected close to the solar panels' level and then reaches about the same direction as the $8.01 \mathrm{eV}$ trajectory (the A16 plane does not cut the solar panels). But between the $7.67 \mathrm{eV}$ trajectory and the aborted $7.33 \mathrm{eV}$ one, there are trajectories reaching infinity with directions very far from the $w_{1}$ window line of sight. For increased values of $\Phi_{s}$ the reflecting effect of the solar panels is even greater. Deviations from the original direction are clearly seen on the $w_{10}$ trajectories and one can also remark that for $w_{16}$, the angular deviations versus energy are in the opposite direction with respect to those relative to the other windows of the figure. In any case the higher the energy is, the smaller the angular deviation.

It seems to be relatively easy to compute angular corrections for the ion fluxes detected by any window for high energies. The energy cutoff will be slightly dependent on the window direction, and ion trajectories at energies just above the cutoff should be difficult to predict.

Under such conditions of ion energies close to the potential barrier energy, we have to reconsider the neglect of the magnetic force. Adding magnetic force in the computation procedure shows effectively that it is always possible to find an initial energy so close to the energy barrier that the ion is quasi-stopped on the barrier and then is highly deviated. But this occurs for an extremely narrow band of energies and this would affect an extremely small number of ions.

As seen above, ions overcoming the potential barrier with a weak energy can be deviated in a wide range of directions. An ion collected through a window $w_{i}$ in an energy channel $E_{j}$ could have any initial (initial for inverse trajectory calculations, but in reality, final for incoming ions) direction in some angular domain and any initial energy within some energy step. The problem is to quantify this effect on ion measurements. In order to do so, we perform numerical simulations of the ion collection on a statistical basis.

\section{Incoming direction probabilities}

\subsection{Procedure}

We have focused our presentation on the A16 analyzer which was systematically used in data treatment, due to its better resolution. However, the same procedures with slightly different parameters can also be used for the A10 analyzer.

We launched a large number of ions (10000 in practice) with a random direction in the solid angle window $\left( \pm 5^{\circ}\right.$ around both central polar and azimuthal angles $\left.\theta_{i}, \phi_{i}\right)$ and a random energy in the energy window $E_{j}$. Then, if the ions reached the outer $30 \mathrm{~m}$ radius sphere simulating infinity, we analysed the number of ions reaching infinity in all directions defined by the polar and azimuthal angles, $\theta_{\infty}$ and $\varphi_{\infty}$, relative to the cartesian frame $X Y Z$. These directions have also been distributed into $10^{\circ} \times 10^{\circ}$ pixels. The fact that we assumed exactly contiguous energy steps simplifies the presentation of the results as two angular dimension maps, since there is a simple obvious energy shift between infinity and the analyzer windows. 
3.2 Results in the case $\Phi_{s}=4 \mathrm{~V}$ and $\Phi_{h}=-4 \mathrm{~V}$

We used again our typical example with $\Phi_{s}=4 \mathrm{~V}$ and $\Phi_{h}=-4 \mathrm{~V}$, focusing our attention on the energy windows close to the energy barrier. The ion distribution in the pixels $\theta_{i \infty}, \phi_{j \infty}$ has been normalised with respect to the total number of ions which reach infinity. The results are reported in Fig. 5 for the 4 energy channels ranging from $4.6 \mathrm{eV}$ to $11.3 \mathrm{eV}$. The $\theta_{\infty}$ range is $\left(0-180^{\circ}\right)$ and the $\varphi_{\infty}$ range is $\left(90-360^{\circ}\right)$, whereas A16 is pointing at $240^{\circ}$. We do not present the lower energy channels which are all well below the cutoff and we do not present the upper channels for which there is a relatively small deviation and no spreading. The horizontal pink bar at the bottom of each plot represents the number of ions reaching infinity normalised to the number of ions launched from each window. For most of the plots the bar length is full scale (not any ion lost). For some plots, the reduced bar length is an indication of the defective "transparency" of the window for the energy step considered.

The windows $\left(w_{1}-w_{8}\right)$ for the first passing energy range of (7.3-9.0 eV) exhibit a remarkable dispersion: (i) at all angles close to the analyzer plane, shifting to the right of the figures, (ii) in a widely $\varphi$-spread structure at the direction which corresponds normally to lower windows. This effect can be understood since the ions which are passing at the top of a potential barrier are very slow here and can be deflected in any direction as we predicted; then, the solar panels' potential structure acts as an electrostatic mirror. The asymmetry of the probability distribution reflects the asymmetry of the Hyperboloid environment linked to the location of the solar panels and to the proximity of the POLRAD antenna. In practice, the eight windows $w_{9}-w_{16}$ exhibit a low $\theta$ dispersion corresponding to a relatively free direction in the potential map (see Fig. 2, lower panel). The wider spreading in azimuth is related to the lens effect of the potential structure between the two adjacent star branches of the upper panel of Fig. 2.

\subsection{Discussion about the incoming probability maps}

Our 2D potential map (see Fig. 2, upper panel) shows the same saddle structure as the potential maps computed by Olsen et al. (1986) around the DE-1 satellite. There is an absolute maximum height of the potential barrier that the ions have to cross in order to reach the instrument, but we deal, in fact, with the maxima of potential reached by the ions along their trajectories from (or to) the detectors. For each window, our statistical treatment allows one to find in the 3D real conditions the energy windows for which all ions are repelled. This energy channel cutoff will be compared later with the measurements.

We have been able to quantify the angular spreading of the ions close to the energy cutoff with our random inverse trajectories' calculations. Again, with 10000 ions with random initial conditions, we introduced a $1.5 \mu \mathrm{T}$ magnetic field in two orthogonal directions. There was no difference on the probability maps. This justifies neglecting the magnetic field effect in our case because we do not require a relative accuracy of flux measurements better than $10^{-4}$.

For higher energies the spreading is reduced and we have deduced the angular deviations in polar angle and in azimuth. However, to be more precise, this treatment should take into account more detailed acceptance characteristics of the detectors: here, we considered sharp windows in energy and angles, whereas the real instrument can collect a few ions of the neighbouring energy range or closely outside the geometrical angular range of the windows. Such a treatment could be performed in a further study. Nevertheless, for the energy channel $E_{j}$ which includes the energy barrier, the angular deviations of ion trajectories depend strongly on the ion energy, as previously shown in Fig. 3. Due to the width of the channel $E_{j}$, it is not possible to determine the incoming direction of ions without more assumptions.

\subsection{Ion beam simulation}

Our probability maps are made without any assumption about the characteristics of the incoming ion distribution. For any ion detected, they define an angular domain from where this ion could come. How can we use these probability maps to treat the direct problem of ions coming from the plasma? It is extremely difficult and computationally costly to proceed to a direct simulation of the ion fluxes from infinity to the detector. So, we made the assumption of a beam of ions incoming from infinity in a $10^{\circ} \times 10^{\circ}$ solid angle, from some polar angle with respect to the spin axis. As the satellite rotates, the windows are either hit or not hit by the ions according to the probability maps. The result is a boolean map as shown in Fig. 6. Two energy steps are considered (7.3$9.0 \mathrm{eV})$ in the left side and $(9.0-11.3 \mathrm{eV})$ in the right side. The 12 maps, from top to bottom, are relative to 12 polar angles ranges of the incident (from infinity) beam: $\left(10^{\circ}-20^{\circ}\right)$ $-\left(120^{\circ}-130^{\circ}\right)$. We consider only these beam polar angle directions between $10^{\circ}$ and $130^{\circ}$ because in the Hyperboloid data, the upward magnetic field line direction lies in this range. Each boolean map represents the possibility for the window $w_{i}$ (16 windows $1-16$ on the vertical axis) to detect an ion from the considered beam at a given time during the rotation of the satellite. For this reason the horizontal axis represents time as well as an azimuthal (i.e. spin) angle in the range $0^{\circ}-360^{\circ}$, whose value is $240^{\circ}$ when the incident beam is in the A16 plane.

These boolean "transparency maps" relative to several incident beam directions do not predict any value of flux but should demarcate the domain of reception of each window during the satellite rotation. For the critical energy step which includes the potential barrier, a dispersion in polar angle (or window number) as well as a dispersion in azimuth (or time) is clearly seen. The deviations in time have to be referred to the time when the beam direction is in the A16 plane (pointed out as "hyp" on the figure). A side "echo domain" is also present before (in time) the main domain for all beam directions in the range $50^{\circ}-130^{\circ}$. As foreseen, for the higher energy step, the dispersion is reduced but the devia- 

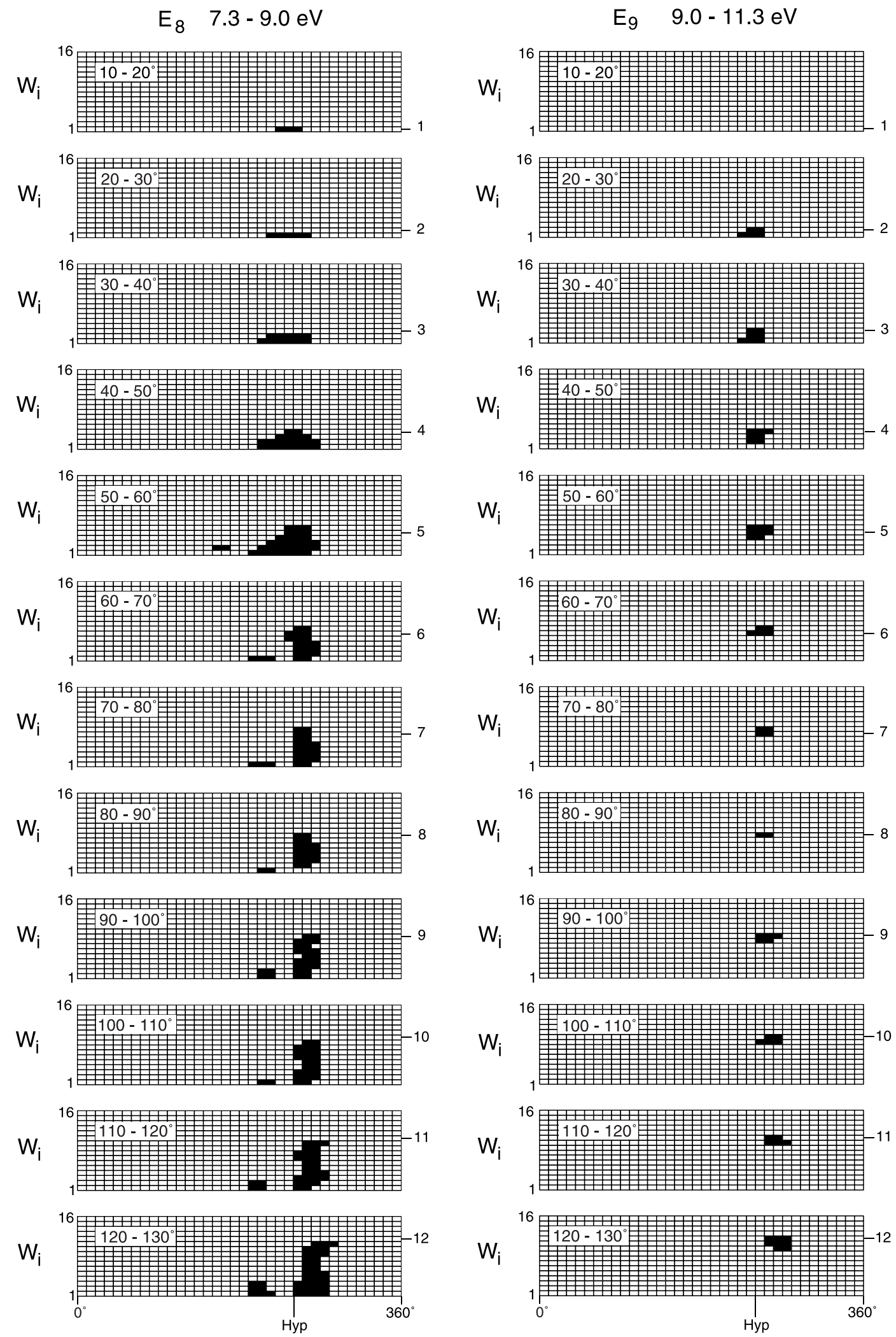

Fig. 6. Simulated maps of the possibility to detect ion beams during a spin period for various incoming beam directions at infinity and for two energy bands: $7.3-9.0 \mathrm{eV}$ (left) and 9.0-11.3 eV (right). From top to bottom, each plot corresponds to an incoming polar angle of the ion beam. Inside each plot, black cells represent the possible arrival directions versus windows $w_{i}$ and azimuthal angle (or time since the satellite is rotating). The number in the right side of each plot denotes the window aligned with the incoming direction of the beam at infinity. The label "HYP" refer to the azimuthal angle of the instrument. 
tions from the incoming directions remain. It is now interesting to compare these simulations to an example of measured data with realistic assumptions on the beam direction.

\section{Application to Hyperboloid data and the "ion double flows"}

We tried to check our predictions with the data recorded at high altitude $\left(2-3 R_{E}\right)$ by the Hyperboloid A16 analyzer. Using probability maps based on particle trajectories in order to analyse low energy ion measurements and correct ion distributions requires the knowledge of the spacecraft potential.

Most of the time this potential can be estimated from electric field double-probe measurements by the IESP experiment (Perraut et al., 1998). This experiment provides the potential difference $\Phi_{s p}$ between the spacecraft body $(s)$ and the probes $(p)$ at the end of electric booms. The inherent difficulty with probe measurements lies in their own electric potential $\Phi_{p}$ with respect to the plasma. Therefore, the IESP experiment does not measure directly the spacecraft potential $\Phi_{s}$ with respect to the plasma, but the difference $\Phi_{s p}=\Phi_{s}-\Phi_{p}$. However, by using the equilibrium of currents flowing in and out of the spacecraft body and the probes, we could numerically derive $\Phi_{s}$ and the ambient plasma density $N_{e}$ as functions of $\Phi_{s p}$. Details of this technique, along with some results, are described in a companion paper (Bouhram et al., 2001). For the typical plasma densities encountered by Interball-2, $\Phi_{p}$ is of the order of $2 \mathrm{~V}$ and depends weakly on the plasma parameters. A statistical analysis of the IESP observations in the polar cap indicated that $\Phi_{s p}$ ranges from $\sim 2$ to $\sim 12 \mathrm{~V}$. The effects on low energy ion measurements linked to the spacecraft charging phenomenon and to the complex potential structure around the satellite should therefore be reflected in polar wind observations.

\subsection{Observations}

The polar wind data, shown in Fig. 7, come from an Interball2 pass through the pre-midnight polar cap at altitudes of $\sim 15000 \mathrm{~km}$, on 21 September 1997. According to Wind observations, this pass occurred during a period of positive IMF $B_{Y}$ component $(\sim 5 \mathrm{nT})$ and negative IMF $B_{Z}$ component $(\sim-4 \mathrm{nT})$. To compare more easily the experimental results with the predictions of Sect. 3, the angular distribution of low energy $\mathrm{H}^{+}$and $\mathrm{O}^{+}$ions is presented through color-coded polar spectrograms. Plotted in the top four panels are $\mathrm{H}^{+}$fluxes averaged over several energy ranges $(0-4 \mathrm{eV} ; 4-6 \mathrm{eV} ; 6-9 \mathrm{eV}$ and $9-15 \mathrm{eV}$ ) as a function of window number, i.e. polar angle (ordinate) versus time, i.e. spin or azimuthal angle (abscissa). Let us recall that each window has an angular width of $10^{\circ} \times 10^{\circ}$. For this data set each scan over energies and polar angles lasts $8 \mathrm{~s}$. The white squares and crosses indicate ions moving downward and upward along the magnetic field lines, respectively. The full white line (cosine structure) cor- responds to $90^{\circ}$ pitch-angle. The upward field-aligned direction lies in the $w_{5}$ window. The spin period $(2 \mathrm{mn})$ modulated red stripes appearing in these spectrograms represent the signature of outflowing ion fluxes. During each spin period, intense fluxes are detected at the same times (21:51:30 UT, 21:53:30 UT,...), i.e at the same azimuthal angles, in the energy range $4-9 \mathrm{eV}$, at polar angles in the $0^{\circ}-50^{\circ}$ range $\left(w_{1}\right.$ to $w_{5}$ windows). This corresponds to ion velocities, as seen by Hyperboloid, between the upward field-aligned direction $\left(w_{5}\right.$ window) and the opposite to the spin axis direction, i.e. the antisunward direction. As we will see later, the corrections linked to the "ram" effect and to the convection velocity are not sufficient to explain the polar angle dispersion observed in the spectrograms. It may be noted that the weak fluxes recorded in the lowest energy channel $(0-4 \mathrm{eV})$ are artificial signatures due to the acceptance effect and induced by the intense fluxes observed in the adjacent energy channel just above $4 \mathrm{eV}$. As mentioned in Sect. 3, fluxes in a channel $E_{j}$ induce fluxes of about two orders of magnitude lower in the adjacent channels $E_{j \pm 1}$. Indeed, as can be seen in the middle panel, the spacecraft potential is almost constant and equal to $\sim 4 \mathrm{~V}$ throughout the selected time period. As for this case study, the negative bias potential relative to the satellite applied to the external cover of the Hyperboloid spectrometer is $-8 \mathrm{~V}$, the energy in the Hyperboloid frame of an ion entering into the spectrometer must be at least $4 \mathrm{eV}$.

A second characteristic feature is apparent in the $\mathrm{H}^{+}$ spectrograms: during each period, a second signature is seen on the spectrograms about 20-40 s (azimuthal shift of $60^{\circ}-120^{\circ}$ ) before the main signature. The corresponding fluxes are weaker (one to two orders of magnitude) and are detected in the $w_{2}$ to $w_{6}$ windows. These outflowing ions are observed at pitch-angles closer to $90^{\circ}$ than the previous ones.

Similar signatures are also observed in the $\mathrm{O}^{+}$ion spectrograms (bottom panels) at the same energies, but clearly before (in spin angle) the $\mathrm{H}^{+}$signatures. The $\mathrm{O}^{+}$main signature is always observed between 8 and $16 \mathrm{~s}$ ( 1 and 2 times swap durations) before the $\mathrm{H}^{+}$main signature (spin angle shift between $24^{\circ}$ and $48^{\circ}$ ). The spin angle gap between the second $\mathrm{O}^{+}$signature (clearly seen in the $6-9 \mathrm{eV}$ energy channel, but less visible between $4 \mathrm{eV}$ and $6 \mathrm{eV}$ ) and the main $\mathrm{O}^{+}$ signature is the same as that observed for $\mathrm{H}^{+}$ions. We note also that, for both ions, the gap between the main and second signatures remains almost constant in time, when taking into account the width of the signatures.

We shall see below that the $\mathrm{O}^{+}$and $\mathrm{H}^{+}$faint signatures observed before the main ones can be qualitatively explained when taking into account the potential structure near the satellite and can be attributed to ions of the polar wind outflow undergoing a strong deviation before reaching the $\mathrm{Hy}$ perboloid instrument.

\subsection{Interpretation and discussion}

The ion velocity measured in the Hyperboloid frame is the sum of the upflow velocity, the ram velocity $\boldsymbol{V}_{\text {ram }}$ (opposite to the spacecraft velocity) and the convection velocity 


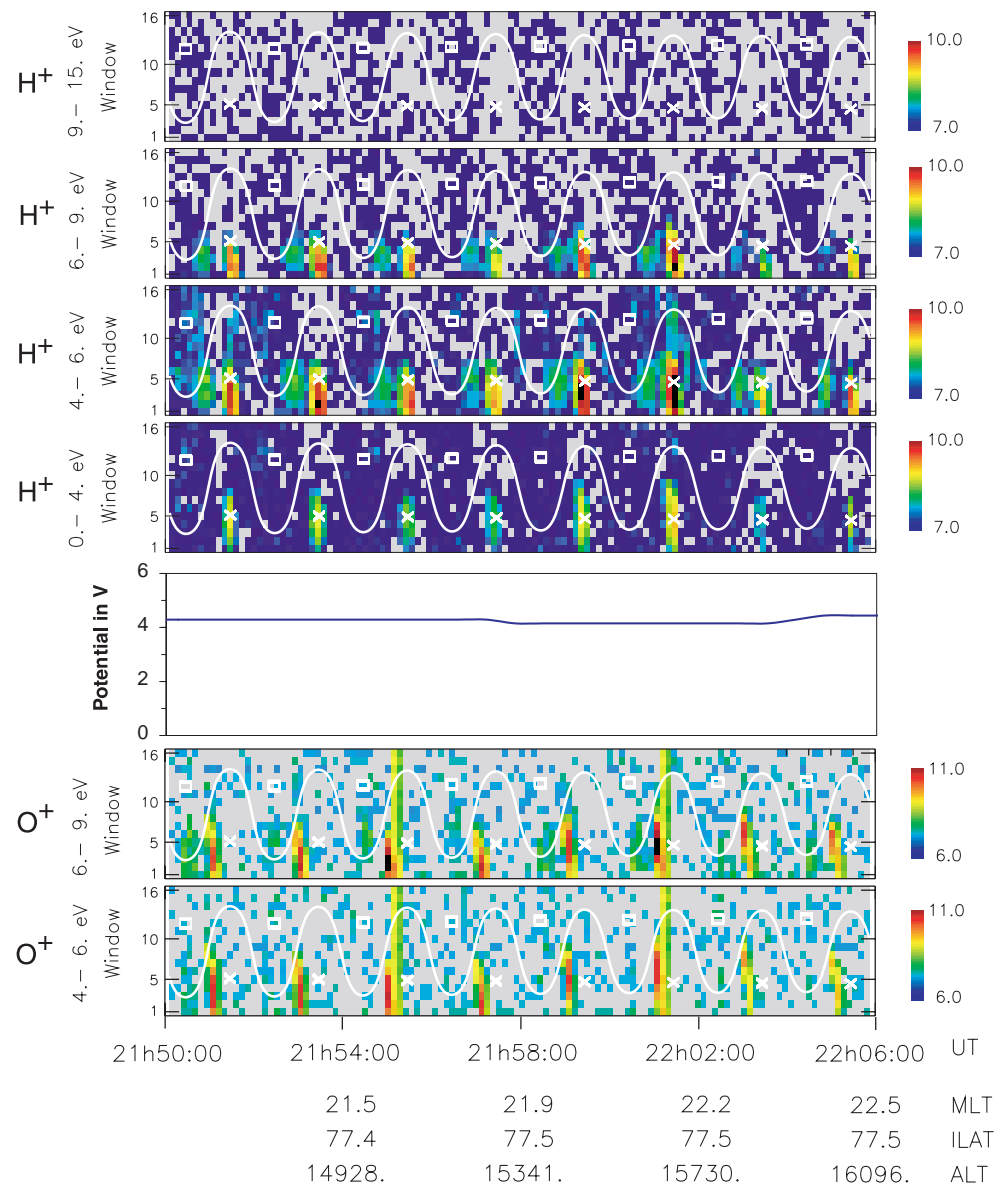

Fig. 7. $\mathrm{H}^{+}$and $\mathrm{O}^{+}$distributions recorded by the Hyperboloid A16 analyser on 21 September 1997 between 21:50 and 22:06 UT. The upper four panels display the angular variation of $\mathrm{H}^{+}$fluxes in $\log \left[\left(\mathrm{m}^{2} \mathrm{~s} \text { sr eV }\right)^{-1}\right]$ for different energy ranges. The middle panel shows the time evolution of the satellite potential, while the bottom two panels display the angular variation of $\mathrm{O}^{+}$fluxes in $\log \left[\left(\mathrm{m}^{2} \mathrm{~s} \text { sr eV }\right)^{-1}\right]$ for different energy ranges.
$\boldsymbol{V}_{\text {conv. }}$ If we consider ions having the same energy, the ram and convection effects are more significant for $\mathrm{O}^{+}$ions than for $\mathrm{H}^{+}$ions. Let us consider measurements made between 21:50:30 UT and 21:52:30 UT to illustrate these effects. The main $\mathrm{H}^{+}$signature is observed during the energy and window scans, occurring around 21:51:30 UT. At this time the upward magnetic field direction lies in the A16 analyzer plane. The convection velocity deduced from $\mathrm{O}^{+}$ ion measurements has a component parallel to the A16 analyzer plane of $\sim 3.7 \mathrm{~km} / \mathrm{s}$ (oriented at $145^{\circ}$ from the spin axis direction) and a smaller component $(0.7 \mathrm{~km} / \mathrm{s})$ in the perpendicular direction (roughly dawn to dusk). For the ram velocity, the components are, respectively, $2.2 \mathrm{~km} / \mathrm{s}$ and $2 \mathrm{~km} / \mathrm{s}$. So the component of $\left(\boldsymbol{V}_{\text {ram }}+\boldsymbol{V}_{\text {conv }}\right)$ perpendicular to the A16 analyzer has a magnitude of $2.7 \mathrm{~km} / \mathrm{s}$, which is not negligible compared to the ion velocity in the upward field-aligned direction for $\mathrm{O}^{+}$ions having a typical energy of $5 \mathrm{eV}$. The correction is very small for $\mathrm{H}^{+}$ions of same energy. This explains the observed spin angle gap between the $\mathrm{O}^{+}$and $\mathrm{H}^{+}$ main signatures. The other components of $\left(\boldsymbol{V}_{\text {ram }}+\boldsymbol{V}_{\text {conv }}\right)$ contribute to the shift in the ion directions of arrival toward windows closer to the spin axis $\left(w_{1}\right.$ to $\left.w_{5}\right)$. The fact that fluxes are recorded simultaneously in several windows over a polar angle range of $\sim 50^{\circ}$ (red stripes in the spectrograms) is partly explained by the temperatures of the ion distribu- tions.

However, the calculations developed in Sect. 3 predict that trajectories of ions having energies just enough to overcome the potential barrier are very sensitive to the initial arrival directions of ions far from the satellite. In our polar wind event, $\mathrm{H}^{+}$ions flow upward along magnetic field lines in a direction determined by a polar angle $\sim 55^{\circ}\left(w_{5}\right.$ window) and the spin angle value $\varphi_{0}$ corresponding to times when the $\mathrm{H}^{+}$main signatures are observed (at these times the magnetic field direction is in the A16 plane). In the Hyperboloid frame, due to combined effects of temperature, ram and convection velocities and potential structure (see the right panels of Fig. 6), these ions, at least those having energies above the potential barrier, are detected in a widened polar angle range $\left(w_{1}\right.$ to $w_{5}$ windows) in a spin angle range $\Delta \varphi$ of $\sim 20^{\circ}$ around $\varphi_{0}$. Regarding ions with energy close but above the potential barrier, Fig. 6 indicates that ions with an arrival direction far from the satellite that lies in the $w_{i}$ window $(i \leq 5)$ have a non-negligible probability of being observed in the windows with smaller indices not only at a spin angle $\sim \varphi_{0}$, but also at smaller spin angles with a shift that can amount up to $50^{\circ}$ for $w_{4}$ and even $90^{\circ}$ for $w_{5}$. These results are qualitatively in agreement with the observations, but we must nevertheless emphasize that the gap between the two signatures is only predicted for arrival directions far from the satellite in the $w_{5}$ 
window. The main prediction is rather a broadening of the spin angle range where ions may be detected. The interpretation of the second $\mathrm{H}^{+}$signature in terms of effects linked to the potential structure is also qualitatively valuable for the second $\mathrm{O}^{+}$signature, since for a given energy, these effects do not depend on ion mass, but only on ion charge and potential barrier (and therefore on satellite potential).

\subsection{Application to correction of ion distribution}

The probability maps calculated with the method developed in Sect. 3 can be used to correct the measured ion distributions. As already mentioned, the positive spacecraft potential has two consequences. First, the potential barrier prevents ions with energies less than the barrier height $E_{\text {bar }}$ from reaching Hyperboloid and therefore a part of the thermal ion distributions can be missed. Second, trajectories of ions with energies just above $E_{\mathrm{bar}}$ are strongly modified in comparison with those in the absence of a barrier. A correction of ion distributions is meaningless if the spacecraft potential varies during the experimental time required to obtain an ion distribution (a spin period). If the spacecraft potential does not vary significantly during a spin period, we can reconstruct the ion distribution proceeding as follows:

- knowing the spacecraft potential and estimating the potential barrier from measurements, we make the angular corrections using the probability maps and reconstitute the corresponding part ( $E>E_{\text {bar }}$ ) of the ion distribution;

- we calculate the moments (density, bulk velocity, parallel and perpendicular temperatures) deduced from this rebuilt distribution and use them as input in an iterative process to estimate the missing part. Of course this procedure presupposes that we have chosen the form of the ion distribution.

In this paper we restrict our study to the first part. The complete method and its application to a statistical analysis of the polar wind observations by Interball- 2 will be the subject of a future paper.

\subsubsection{Method}

For a given ion species, measurements are performed by Hyperboloid at times $t=t_{k}$ (spin angle $\varphi_{k}$ ) during a set of successive swaps over energy channels $E_{j}$ and windows $w_{i}$, forming a three-dimensional array of ion fluxes $J_{i, j, k}$. Since Hyperboloid rotates around the spin axis, it took a spin period to cover all directions relative to $\boldsymbol{B}_{0}$ and to construct a $3 \mathrm{D}$ velocity distribution. The ion phase-space density $F_{i, j, k}$ can be derived from ion fluxes using the formula:

$F_{i, j, k}=J_{i, j, k} \times M^{2} /\left(2 q^{2} E_{j}\right)$,

where $q$ and $M$ denote the ion charge and mass, respectively, and $E_{j}$ is the measured ion energy in the channel $j$.
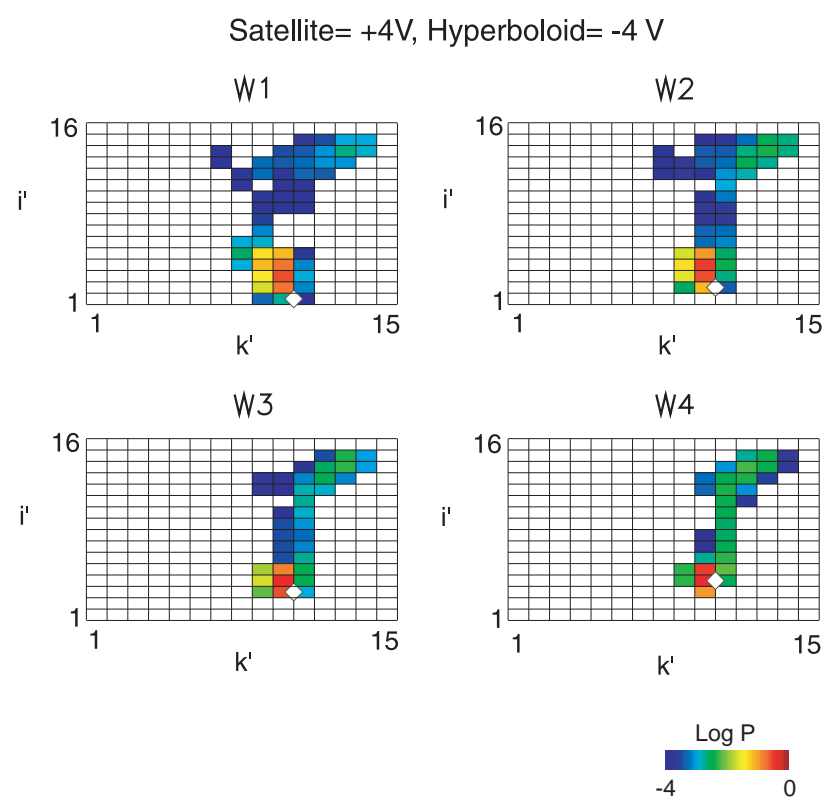

Fig. 8. Incoming direction probabilities for windows 1 to 4 in the energy range 6.0-9.0 eV used in correcting ion distributions. Maps are interpolated, the new angular resolution is $24^{\circ} \times 10^{\circ}$ pixels according to the data resolution.

By setting the spacecraft potential to a value $\Phi_{s}$, we have described in Sect. 3 a method of determining, for each couple $(i, j)$ of window $w_{i}$ and energy channel $E_{j}$, a probability map of incoming directions $P_{i, j}\left(\theta_{\infty}, \varphi_{\infty}\right)$. Providing $\Phi_{s}$ is determined in-flight from IESP measurements and assuming a steady state ion distribution during a spin period, such probability maps can be used for angular corrections on the measured distributions. It must be stressed here that we made a priori no assumption about the shape of the incoming ion distributions. The inferred probability maps $P_{i, j, m, n}=P_{i, j}\left(\theta_{\infty m}, \varphi_{\infty}\right)$ have an angular resolution of about $10^{\circ} \times 10^{\circ}$. Such a resolution is the highest angular resolution of the operating modes of the Hyperboloid instrument. For the data described in Fig. 7, the angular resolution is about $10^{\circ} \times 24^{\circ}$, and probability maps have been interpolated accordingly. Figure 8 shows the interpolated maps $P_{i, j, i^{\prime}, k^{\prime}}$ for $i$ ranging from 1 to 4 .

Let us consider a phase-space measurement $F_{i, j, k}$ at a time $t=t_{k}$, in the window $w_{i}$ and in the energy channel $E_{j}$. We can associate with this measurement $F_{i, j, k}$ a probability map $P_{i, j}\left(\theta_{\infty}, \varphi_{\infty}\right)$. If a probability $P_{i, j, i^{\prime}, k^{\prime}}$ is non-zero, it should induce a contribution $P_{i, j, i^{\prime}, k^{\prime}} \times F_{i, j, k} \times\left(\sin \theta_{i} / \sin \theta_{i^{\prime}}\right)$ to the measured phase-space density in the window $w_{i^{\prime}}$ at a time $t=t_{k^{\prime}}$ defined by:

$t_{k^{\prime}}=t_{k}+\frac{T}{360} \times\left(\varphi_{k^{\prime}}-\varphi_{H}\right)$,

where $\varphi_{H}$ is the azimuthal angle of Hyperboloid relative to the frame used. Since the phase-space volumes for the cells $(i, j, k)$ and $\left(i^{\prime}, j, k^{\prime}\right)$ are different, a factor $\left(\sin \theta_{i} / \sin \theta_{i^{\prime}}\right)$ appears when applying Liouville's theorem. We note that en- 

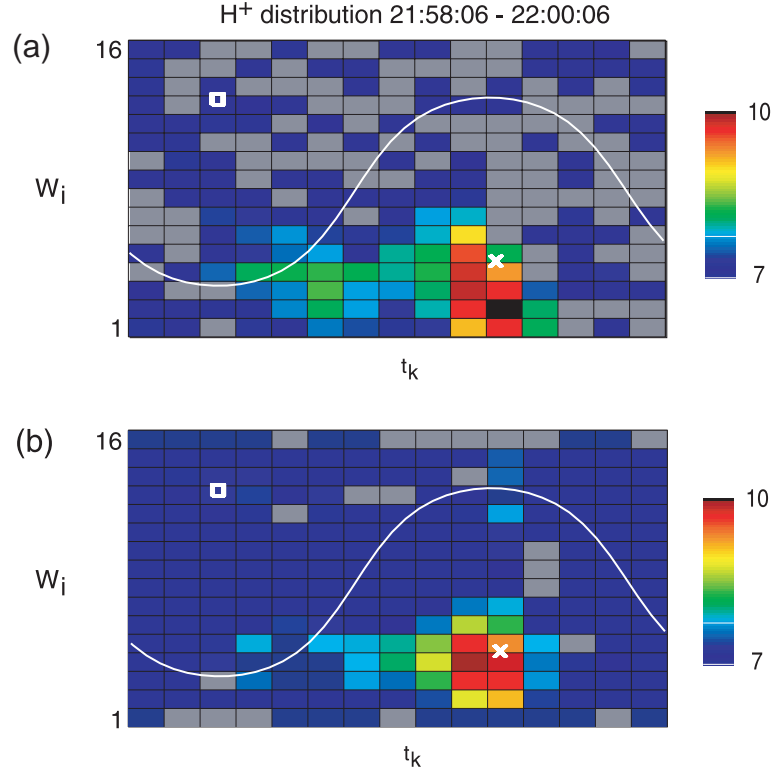

Fig. 9. An example of angular corrections: (a) $\mathrm{H}^{+}$angular distribution observed between 21:58:06 and 22:00:06 UT at measured energies ranging from 6.0 to $9.0 \mathrm{eV}$, (b) the corresponding corrected distribution.

ergies of ions reaching Hyperboloid are shifted by the same amount of energy $-q \Phi_{h}$, regardless of the initial energies at infinity. Therefore, angular corrections can be made separately from energy corrections. The phase-space density $F_{i^{\prime}, j, k^{\prime}}$ at a time $t=t_{k^{\prime}}$ is found after applying the corrections over all windows and time steps, and can be expressed as:

$F_{i^{\prime}, j, k^{\prime}}=\sum_{k} \sum_{i} P_{i, j, i^{\prime}, k^{\prime}} \times F_{i, j, k} \times\left(\sin \theta_{i} / \sin \theta_{i^{\prime}}\right)$.

\subsubsection{Results}

Figure 9 shows an example of measured distribution before and after correcting angular disturbances for the energy channel (6-9 eV in the Hyperboloid frame, $2-5 \mathrm{eV}$ at infinity) including the potential barrier. The correction reduces the dispersion in polar and spin angles and leads to a distribution much more aligned with the upward magnetic field direction as expected for a polar wind ion population. This distribution is not fully gyrotropic yet, since ram and convection corrections have not been included. The second signature vanishes, which confirms the theory that it is the result of the interaction between the ions and the potential around the spacecraft.

\section{Conclusion}

We have shown that for Hyperboloid on Interball-2, as well as for DE-1, the biasing technique proved to be not very efficient in reducing the potential barrier; in consequence the truncation of the energy distribution is not avoided. The correction of measurements of the energy and angle thermal ion distributions gives valuable and reliable information on the accessible part of the distribution at the cost of a complex data analysis. We have shown how, from estimations of the satellite potential, it was possible to deduce probability maps in order to correct and analyze the angular distributions in the unperturbed plasma. Such corrections are necessary for studying characteristics of the polar wind, as discussed in Sect. 4. The estimation of the missing part of the ion distribution requires reliable information about the angular distribution for energies above the potential barrier. Taking into account only energy corrections could induce errors in the rebuilt ion distributions.

Our results emphasize the difficulties associated with measuring the ion thermal plasma. Until now, two methods have been applied for shrinking the satellite potential. The first is to apply a negative bias to the ion detector. As shown here and in previous other experiments, this method is not fully efficient. The second method consists of an active control of the satellite potential by an ion source. Such a technique is effective, but generates electromagnetic perturbations, prejudicial to other experiments aboard the satellite. As already suggested by Olsen (1982), a way to get rid of the energy barrier would be to install the ion detector (as small as possible) on a boom far enough from the satellite body. Under these conditions, the biasing technique should be more effective.

Acknowledgements. The Interball Project was accomplished in the frame of contract N025-7535/94 with the Russian Space Agency (RKA). The Hyperboloid experiment was financially supported by CNES under the auspices of grants covering the period 1985 to 2001.

The Editor in Chief thanks P. Escoubet and another referee for their help in evaluating this paper.

\section{References}

Bouhram, M., Dubouloz, N., Hamelin, M., Grigoriev, S. A., Malingre, M., Torkar, K., Galperin, Y., Hanasz, J., Perraut, S., Schreiber, R., and Zinin, L. V.: Electrostatic interaction between Interball-2 and the ambient plasma. 1. Determination of the spacecraft potential from current calculations, Ann. Geophysicae, this issue, 2001.

Décreau, P. M. E., Etcheto, J., Knott, K., Pedersen, A., Wrenn, G. L., and Young, D. T.: Multi-experiment determination of plasma density and temperature, Space Sci. Rev., 22, 633-645, 1978.

Dubouloz, N., Berthelier, J. J., Malingre, M., Girard, L., Galperin, Yu., Covinhes, J., Chugunin, D., Godefroy, M., Gogly, G., Guérin, C., Illiano, J.-M., Kossa, P., Leblanc, F., Mularchik, T., Paris, J., Stzepourginski, W., Vivat, F., and Zinin, L.: Thermal ion measurements on Interball Auroral Probe by the Hyperboloid experiment, Ann. Geophysicae, 16, 1070-1085, 1998.

Girard, L., Berthelier, J. J., Covinhes, J., Godefroy, M., Gogly, G., Guillou, J., Illiano, J. M., Leblanc, F., Le Goff, F., Stepourginski, W., Vivat, F., Mularchik, T. M., Galperin, Yu. I., Gladyshev, V. A., and Zinin, L. V.: Hyperboloid: the low ion massspectrometer on the INTERBALL auroral probe, in: Interball 
Mission and Payload, (Eds) Galeev, A. A., Galperin, Yu. I., and Zelenyi, L. M., CNES-IKI-RSA, 1995.

Olsen, R. C.: The hidden ion population in the magnetosphere, J. Geophys. Res., 87 (A5), 3481-3488, 1982.

Olsen, R. C., Chappell, C. R., and Burch, J. L.: Aperture plane potential control for thermal ion measurements, J. Geophys. Res., 91, 3117-3129, 1986.

Pedersen, A.: Solar wind and magnetosphere plasma diagnostics by spacecraft electrostatic potential measurements, Ann. Geophysicae, 13, 118-129, 1995.

Perraut, S., Roux, A., Darrouzet, F., de Villedary, C., Mogilevsky, M., and Lefeuvre, F.: ULF wave measurements onboard the In- terball auroral probe, Ann. Geophysicae, 16, 1105-1116, 1998

Torkar, K., Jeszenski, H., Veselov, M. V., Perraut, S., Dubouloz, N., Escoubet, C. P., and Galperin, Yu. I.: Spacecraft potential measurements on board Interball-2 and derived plasma densities, Cosmic Res., 37, 606, 1999.

Zinin, L. V., Galperin, Yu. I., Grigoriev, S. A., and Mulyarchik, T. M.: On measurements of polarization jet effects in the outer plasmasphere, Cosmic Research, 36, 42-52, 1998.

Zinin, L. V., Galperin, Yu. I., Gladyshev, V. A., Grigoriev, S. A., and Mulyarchik, T. M.: Modelling of the anisotropic thermal plasma measurements of the energy-mass angle ion spectrometers onboard a charged satellite, Cosmic Research, 33, 511-518, 1995. 